\title{
The ESEM as In Situ Platform for the Study of Gas-Solid Interactions
}

Jing Cao ${ }^{1}$, Zhu-Jun Wang ${ }^{1}$, Xing Huang ${ }^{1}$, Ali Rinaldi ${ }^{2}$, Mark Greiner ${ }^{3}$, Grigore Moldovan ${ }^{4}$, Wolfgang Joachimi $^{4}$, Robert Schlögl ${ }^{1,3}$ and Marc Willinger ${ }^{1,5}$

1. Department of Inorganic Chemistry, Fritz Haber Institute of the Max Planck Society, Berlin, Germany.

2. Chemistry Department, King Fahd University of Petroleum \& Minerals, Dhahran, Saudi Arabia.

3. Max Planck Institute for Chemical Energy Conversion, Mülheim an der Ruhr, Germany

4. point electronic GmbH, Erich-Neuß-Weg 15, Halle, Germany.

5. Scientific Center for Optical and Electron Microscopy-ScopeM, ETH Zürich, Zürich, Switzerland.

In order to understand the working principle of functional materials they should, at one point, be studied in their working state. In view of electron microscopy, atomic motion and chemical dynamics can be observed by in situ TEM. However, size constrains and the requirement of electron beam transparency impose substantial limitations with respect to dimension, complexity and preparation of a specimen. Furthermore, atomistic details can only be resolved under conditions where atomic scale dynamics are slow compared to the temporal resolution of the detection system. In the case of gas-solid interactions, in situ TEM observations are therefore often performed at reduced chemical potential of the reactive gas phase. Due to the strong focus on ultimate spatial resolution, the potential of environmental scanning electron microscopy (ESEM) as a flexible tool for in situ studies in the field of material science has recently been overlooked.

In situ experiments performed in the ESEM can be used to complement localized information that is obtained by in situ TEM. Instead of atomistic details, it reveals the complexity of hierarchical multi-scale processes in which collective movements of a large number of atoms are involved. Thus, effects related to heat and mass transport are accessible. Compared to the TEM, the ESEM imposes far less restrictions with respect to available space and dimensions of the sample. This opens up the possibility to bridge the "materials gap" between simplified model- and complex real-world system. Similarly, the focus on collective dynamics allows observations at higher chemical potential of the reactive species and thus, a closing of the so-called "pressure gap". Another important aspect is the reduced areal dose rate and lower kinetic energy of the beam electrons in ESEM. Contributions and extent of various beam induced processes are different and, in most cases, less severe than in the TEM. Furthermore, the ESEM allows fast and efficient screening of the parameter field and facilitates a more efficient use of the in situ TEM set-up.

In order to obtain a unique platform for in situ studies of gas-solid interactions under controlled atmosphere, we have equipped a commercial ESEM with a home-built gas-feeding station, a heating stage, and a mass spectrometer. For topography and 3D imaging of surface dynamics at temperatures of up to $1000{ }^{\circ} \mathrm{C}$, a newly developed four-quadrant BSE detector was implemented. In addition, a detector for electron beam absorbed current (EBAC) was installed in order to complement the large-field detector with a signal that is independent of gas composition and pressure.

It will be shown how the use of this instrument allows to study the emergence of catalytic function in the interplay between a gas-phase and a metal catalyst [1]. Dynamics of metal catalysts under redox conditions will be presented (Figure 1a, b) as well as the ability to study vapour-liquid-solid growth of semiconductor nanowires (Figure 1c, d) [2]. Due to the high sensitivity of the SE signal, it is even possible to study metal 
catalysed chemical vapour deposition of graphene at $1000{ }^{\circ} \mathrm{C}$ (Figure 1e) [3,4]. Finally, it will be demonstrated that contrast variations due to different molecular species on the surface of platinum during catalytic $\mathrm{NO}_{2}$ hydrogenation can be detected (Figure 1f).

References:

[1] M. Greiner et al, Phys.Chem.Chem.Phys. 17 (2015), p. 25073.

[2] X. Huang et al, Adv. Funct. Mater. 25 (2015), p. 5979.

[3] Z.-J. Wang et al, ACS Nano 9 (2015), p. 1506.

[4] Z.-J. Wang et al, Nature Communications 7 (2016).

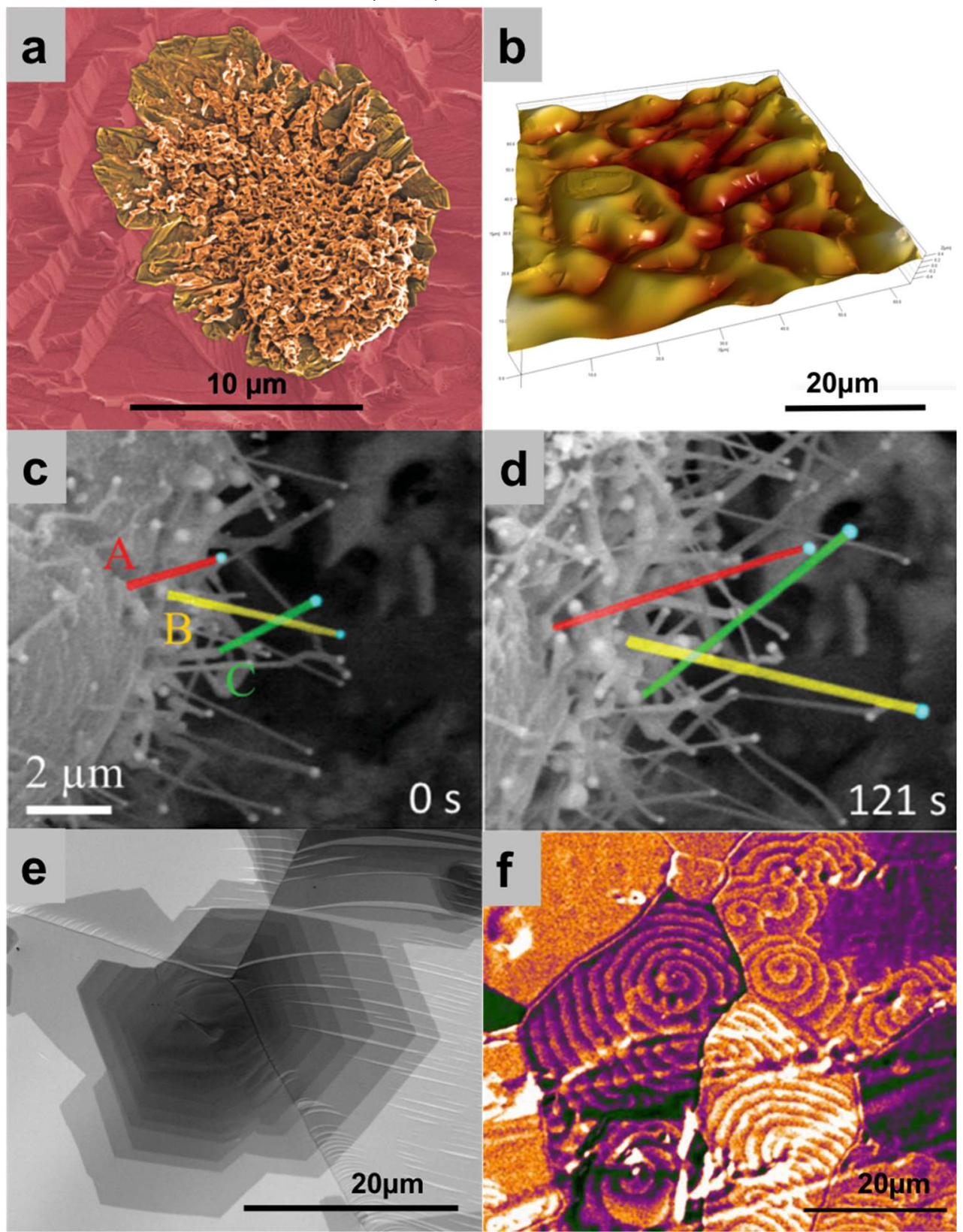

Figure 1. (a): oxidation of $\mathrm{Cu}_{2} \mathrm{O}$ to $\mathrm{CuO}$. (b): topography of a $\mathrm{Cu}$ catalyst during hydrogen oxidation. (c)-(d): vapor-liquid-solid growth of $\mathrm{ZnS}$ nanowires from $\mathrm{CdS}$ and $\mathrm{ZnS}$ with $\mathrm{Ag}$ catalyst particles. (e): few-layer graphene growing on a Pt substrate at $1000{ }^{\circ} \mathrm{C}$ during chemical vapor deposition growth. (f): spiral waves formed by the propagation of reaction-diffusion fronts in the hydrogenation of $\mathrm{NO}_{2}$ on a polycrystalline $\mathrm{Pt}$ foil. Contrast in the colorized image is due to different absorbed molecular species. 